\title{
A multicenter, prospective, randomized study protocol to demonstrate the superiority of a bone-anchored prosthesis for anular closure used in conjunction with limited discectomy to limited discectomy alone for primary lumbar disc herniation
}

\author{
Peter Douglas Klassen ${ }^{1} *$, Robert Hes $^{2}$, Gerrit Joan Bouma ${ }^{3}$, Sandro Eustacchio ${ }^{4}$, \\ Martin Barth $^{5}$, Adisa Kursumovic ${ }^{6}$, Senol Jadik ${ }^{7}$, Volkmar Heidecke ${ }^{8}$, Richard Bostelmann ${ }^{9}$, \\ Claudius Thomé $^{10}$, Peter Vajkoczy ${ }^{11}$, Hans-Peter Köhler ${ }^{12}$, Javier Fandino ${ }^{13}$,Richard Assaker ${ }^{14}$, \\ Erik Van de Kelft ${ }^{15}$, Susanne Fröhlich ${ }^{16}$, Wimar van den Brink ${ }^{17}$, Jason Perrin ${ }^{18}$, \\ Jasper Wolfs ${ }^{19}$, Mark Arts ${ }^{20}$, Frederic Martens ${ }^{21}$
}

Department of Neurosurgery, ${ }^{1}$ St. Bonifatius Hospital, Lingen, Germany, ${ }^{2}$ AZ Klina, Brasschaat, Belgium, ${ }^{3}$ Sint Lucas-Andreas Ziekenhuis and Academic Medical Center, Amsterdam, The Netherlands, ${ }^{4}$ Medical University Graz, Graz, Austria, ${ }^{5}$ University Hospital, Bochum, Germany, ${ }^{6}$ Deggendorf, Germany, ${ }^{7}$ University Hospital, Kiel, Germany, ${ }^{8}$ Klinikum Augsburg, Augsburg, Germany, ${ }^{9}$ University Hospital, Düsseldorf, Germany, ${ }^{10}$ Innsbruck Medical University, Innsbruck, Austria, ${ }^{11}$ Charité Universitätsmedizin, Berlin, Germany, ${ }^{12}$ Asklepios Westklinikum Hamburg, Hamburg, Germany, ${ }^{13}$ Kantonsspital Aarau, Aarau, Switzerland, ${ }^{14}$ Centre Hospitalier Régional Universitaire de Lille, Lille, France, ${ }^{15}$ AZ Nikolaas, Sint-Niklaas, Belgium, ${ }^{16}$ Department of Orthopedics, Orthopädische Klinik und Poliklinik der Universität Rostock, Rostock, Germany, ${ }^{17}$ Neurochirurgisch Centrum Zwolle, Zwolle, The Netherlands, ${ }^{18}$ Department of Neurosurgery, Universitätsmedizin Mannheim, Mannheim, Germany, ${ }^{19} \mathrm{MCH}$ Westeinde, Den Hague, The Netherlands, ${ }^{20} \mathrm{MCH}$ Antoniushove, Leidschendam, The Netherlands, ${ }^{21}$ OLV Ziekenhuis, Aalst, Belgium

Received: 11 July 2016

Accepted: 29 July 2016

\section{*Correspondence:}

Dr. Peter Douglas Klassen,

E-mail: peterdouglas.klassen@bonifatius-lingen.de

Copyright: (c) the author(s), publisher and licensee Medip Academy. This is an open-access article distributed under the terms of the Creative Commons Attribution Non-Commercial License, which permits unrestricted non-commercial use, distribution, and reproduction in any medium, provided the original work is properly cited.

\section{ABSTRACT}

Background: Same-level reherniation and progressive degeneration with disc height loss are main causes of poor outcome after discectomy and may necessitate reoperation. A novel prosthesis for anular closure was developed to address these causes.

Methods: The design of a multicenter, prospective, randomized, post-market superiority trial comparing limited lumbar discectomy augmented with this device (intervention group) with limited lumbar discectomy alone (control group) is presented.

Results: Patients with single-level (L1-S1) posterior or posterolateral disc herniation and radiologic confirmation of neural compression for whom at least six weeks of conservative treatment has failed are eligible. Patients must have posterior disc height $\geq 5 \mathrm{~mm}$ at index level and baseline Oswestry and VAS leg pain scores of $\geq 40 / 100$. Intraoperatively, subjects meeting anular defect size criteria post-discectomy (4-6 mm tall and 6-10 mm wide) will be randomized to study groups in a 1:1 ratio using centralized, web-based software. A Bayesian statistical approach will be used to enroll 400 to 800 subjects who will be followed for at least 24 months. Two co-primary endpoints will be assessed at 24 months: 1) a composite of leg pain, clinical function, disc height maintenance, and absence of reherniation, reoperation, and device failure; and 2) absence of reherniation based upon independent radiologic analysis.

Conclusions: This type of analysis is becoming increasingly important as governments and health insurers continue to be pressured to spend limited healthcare funding wisely.

Keywords: Disc herniation, Reherniation, Anular closure, Discectomy 


\section{INTRODUCTION}

Despite high rates of safety and success in relieving pain and improving function, up to $30 \%$ of discectomy patients experience unsatisfactory results. ${ }^{1-8}$ Same-level recurrent disc herniation and progressive degeneration with loss of disc height are the most common causes of poor outcome and may require reoperation. ${ }^{5,7,9-15}$ The incidence of recurrent disc herniation reported in literature ranges between $0 \%$ and $27 \%$. $^{1,4,7,9,10,12,13,15-22} \mathrm{~A}$ major risk factor for recurrence is anular defect size observed at time of surgery, with defect widths greater than $6 \mathrm{~mm}$ identified as being at particularly high risk. ${ }^{13,18}$ The majority of patients lose more than $25 \%$ of preoperative disc height after surgery; this loss has been associated with poor clinical outcomes, particularly low back pain. ${ }^{5,9,12,13}$ A novel bone-anchored prosthesis for anular closure was developed in an effort to address these complications. It is hypothesized that the device will minimize the risk of recurrent sciatica and disc herniation by blocking the defect in the disc anulus. The device has been CE-marked since 2009, and initial clinical results have been reported at various conferences. The current post-market study, whose design is the subject of this report, compares limited posterior lumbar discectomy with and without this device.

\section{Study goals and objectives}

The goal of this study is to demonstrate the superiority of limited discectomy augmented with a bone-anchored prosthesis for anular closure compared with limited discectomy alone in preventing recurrent pain, dysfunction, and herniation. Success of each subject and overall study success will be evaluated at 24 months according to the co-primary endpoints defined below:

1. A composite of safety and effectiveness. To be considered a success, a subject must achieve success as follows:

- 15/100-point improvement in Oswestry Disability Index (ODI) score compared with baseline;

- 20/100-point improvement in visual analog scale (VAS) leg pain score compared with baseline;

- Maintenance of at least $75 \%$ of baseline disc height;

- No deterioration of neurological status (femoral stretch or straight leg raise (SLR); motor; reflex; and sensory) at the index level. Mixed neurological outcomes will be adjudicated by the data safety monitoring board (DSMB).

- Radiologic confirmation of device integrity (not fractured or disassembled) and lack of migration (intervention group only). Migration is defined as the presence of anteroposterior (AP) or lateral motion of the device $\geq 2 \mathrm{~mm}$ relative to its initial position, and/or motion of the radiopaque marker(s) beyond the margin of the disc space, associated with extrusion of the occlusion component through the anulus.

- No spontaneous fusion;

- No reherniation at the index level (see definition below); and

- No secondary surgical interventions at the index level.

2. Reherniation. To be considered a success, a subject must not exhibit evidence of recurrent herniation at the index level (on either side) at any time previous to, and including, the 24 month follow up evaluation. Recurrent herniation may be determined surgically by the investigator or radiologically through independent analysis (unless surgical confirmation that the suspected herniation was not an actual herniation, e.g., scar tissue).The intervention group will be determined to be superior to the concurrently randomized control group regarding safety and effectiveness if the rates of overall success are statistically superior for the intervention group compared with the control group for both endpoints. In addition, safety will be evaluated through a comparison of the type(s) and rate(s) of occurrence of adverse events (AEs) between the two groups.

\section{Study design}

This study is a multicenter, prospective, randomized, post-market superiority trial designed to demonstrate superiority of limited posterior discectomy augmented with the bone-anchored prosthesis for anular closure (intervention group) compared with limited posterior discectomy alone (control group) in preventing recurrent pain, dysfunction, and herniation. Randomization is $1: 1$ and occurs intraoperatively, following completion of the limited discectomy, to ensure homogeneity between study groups. The trial is registered at the United States National Institutes of Health Clinical Trials Registry (Identifier: NCT01283438), which may be accessed online at http://www.clinicaltrials.gov. Patients between 21 to 75 years with posterior or posterolateral disc herniations at one level between $\mathrm{L} 1$ and $\mathrm{S} 1$; radiculopathy with a positive SLR [23] (L4/5, L5/S1) or femoral stretch test (L1/2, L2/3, L3/4); radiologic confirmation of neural compression; baseline ODI and VAS leg pain scores of at least 40/100; and six weeks of failed conservative treatment are eligible for enrollment. Comprehensive listings of the inclusion and exclusion criteria are provided in Tables 1 and 2. All investigators must complete the device manufacturer's training program prior to study participation to gain familiarity with the surgical technique for implantation of the device. To minimize learning curve bias, each investigator must have performed or participated in at least three limited posterior lumbar discectomies augmented with the prosthesis for anular closure prior to study participation. Study recruitment was initiated in December 2010, with a completed enrollment goal of approximately three years and planned follow-up of at least two years on all subjects. 
Table 1: Inclusion criteria.

\section{Inclusion Criteria}

1. Age 21 to 75 years and skeletally mature (male or female);

2. Posterior or posterolateral disc herniations at one level between L1 and S1 with confirmation of neural compression using MRI.

[Note: Intraoperatively, only post-discectomy anular defects between 4 and $6 \mathrm{~mm}$ tall and 6 and $10 \mathrm{~mm}$ wide shall qualify];

3. At least six weeks of failed, conservative treatment prior to surgery, including physical therapy, use of antiinflammatory medications at maximum-specified dosage, and/or administration of epidural/facet injections;

4. Minimum posterior disc height of $5 \mathrm{~mm}$ at the index level;

5. Radiculopathy (with or without back pain) with positive straight leg raise (0 - 60 degrees) (L4/5, L5/S1) or femoral stretch test (L1/2, L2/3, L3/4 only);

6. Oswestry Disability Index score of at least 40/100 at baseline;

7. Visual analog scale leg pain (one or both legs) score of at least $40 / 100$ at baseline; and

8. Psychosocially, mentally, and physically able to fully comply with the clinical protocol and willing to adhere to follow-up schedule and requirements.

Table 2: Exclusion criteria.

\section{Exclusion Criteria}

1. Spondylolisthesis grade II or higher ( $25 \%$ slip or greater);

2. Requires spinal surgery other than a discectomy (with or without laminotomy) to treat leg/back pain (scar tissue and osteophyte removal is allowed);

3. Back or non-radicular leg pain of unknown etiology;

4. Prior surgery at the index lumbar vertebral level;

5. Patients with a SCORE of 6 or greater and a subsequent spine DXA T-score less than -2.0 at the index level. For herniations at L5/S1, the average T-score of L1-L4 shall be used;

6. Clinically compromised vertebral bodies in the lumbosacral region due to any traumatic, neoplastic, metabolic, or infectious pathology;

7. Pathologic fractures of the vertebra or multiple fractures of the vertebra or hip;

8. Scoliosis of greater than 10 degrees (both angular and rotational);

9. Any metabolic bone disease;

10. Active infection, either systemic or local;

11. Cauda equine syndrome or neurogenic bowel/bladder dysfunction;

12. Severe arterial insufficiency of the legs or other peripheral vascular disease (Screening on physical examination for subjects with diminution or absence of dorsalis pedis or posterior tibialis pulses. If diminished or absent by palpation, then an arterial ultrasound is required with vascular plethysmography. Absolute arterial pressure below $50 \mathrm{~mm} \mathrm{Hg}$ at the calf or ankle level results in exclusion.);

13. Significant peripheral neuropathy, defined as Type I or II diabetes or similar systemic metabolic condition causing decreased sensation in a stocking-like or non-radicular and non-dermatomal distribution in the lower extremities;

14. Insulin-dependent diabetes mellitus;

15. Morbidly obese, defined as a body mass index $>40$ or weighing more than 100 lbs over ideal body weight;

16. Active hepatitis, AIDS, or HIV;

17. Rheumatoid arthritis or other autoimmune disease;

18. Known allergy to titanium, polyethylene, or polyester materials;

19. Baseline MRI cannot be obtained;

20. Pregnant or interested in becoming pregnant in the next three years;

21. Active tuberculosis or history of tuberculosis in the past three years;

22. History of active malignancy, defined as any invasive malignancy, except non-melanoma skin cancer, unless treated with curative intent with no signs or symptoms of malignancy for at least two years;

23. Immunologically suppressed, defined as receiving steroids for more than one month over the past year;

24. Current anticoagulation therapy other than aspirin, unless anticoagulation therapy may be suspended for surgery;

25. Current chemical/alcohol dependency or significant psychosocial disturbance;

26. Life expectancy of less than three years;

27. Current involvement in active spinal litigation;

28. Current involvement in another investigational study;

29. Incarceration; and

30. Any contraindication for MRI or CT scan (e.g., claustrophobia, contrast allergy). 


\section{METHODS}

\section{Screening and preoperative assessment}

\section{Screening}

All subjects who meet the entry criteria will be considered for inclusion. Any subject meeting one or more of the exclusion criteria will not be permitted to participate in the trial. After informed consent is obtained, subjects will be assigned a study patient identification number. Subjects may become screen failures at any step until randomization occurs, at which point the subject will be considered enrolled. No further study-related follow-up evaluations will be required for non-randomized subjects.

\section{Radiologic assessment}

Within three months prior to surgery, magnetic resonance imaging (MRI) with $\mathrm{T} 1$ and $\mathrm{T} 2$ weighted axial and sagittal images and low-dose, multiplanar computed tomography (CT) at the index level only must be performed. Disc degeneration and extent of disc herniation are assessed by MRI; the pre-existing state of the vertebral bodies is documented by CT. Within 60 days prior to surgery, neutral AP, lateral, and flexionextension radiographs must be obtained to determine baselines for disc height and range of motion at the involved level.

\section{Medical history}

Within 30 days prior to surgery, demographic information will be collected; a detailed medical history, including documentation of prior treatments for back and leg pain, will be obtained; a physical examination will be conducted; and work status, current pain medications, and other drug therapies will be recorded. All patients will complete the simple calculated osteoporosis risk (SCORE) validated risk assessment tool for osteoporosis. ${ }^{24,25}$ Patients with a SCORE of 6 or greater will undergo a subsequent dual energy $\mathrm{x}$-ray absorptiometry (DXA) scan to evaluate bone mineral density. A pregnancy test will be performed for all female subjects of childbearing potential.

\section{Clinical assessment}

Within 30 days prior to surgery, subjects will undergo the following pain and function assessments:

1) Oswestry Disability Index..$^{26}$

2) Visual Analog Scales for back and right and left leg pain using a $100 \mathrm{~mm}$ scale; and

3) SF-36v2 $2^{\text {TM }}$ Health Survey. ${ }^{27,28}$

The baseline scores of the above assessments will be compared with postoperative scores.

\section{Neurological evaluation}

Within 30 days prior to surgery, femoral stretch (L1/2, L2/3, L3/4) or SLR (L4/5, L5/S1), reflex, motor muscle strength, and sensory neurological evaluations will be performed. These results later will be compared with those obtained postoperatively.

\section{Surgery and randomization}

All subjects will be prepared for surgery according to hospital and investigator protocol and a single-level, limited discectomy as described by Spengler will be performed. ${ }^{29}$ This technique will remove any nucleus that has migrated within the anular defect or beyond the anular wall, including sequestered fragments. Loose fragments of nucleus from within the disc will be removed in subjects with extrusions or protrusions. Any nuclear material removed, either from within or outside the disc, will be placed dry in a syringe and the volume will be measured and recorded. After completion of the discectomy, the size of the anular defect will be measured and recorded. If the defect is between 4- and 6-mm tall and 6- and 10-mm wide, the subject will qualify for randomization in the study. Any subject whose defect size does not meet this requirement will not be considered enrolled, but will have data collected and reported through the day of surgery.

Subjects who meet the intraoperative criteria will be randomized to study groups in a $1: 1$ ratio using a computer-generated randomization scheme maintained by a centralized randomization center, at which point no further removal of nucleus is allowed. Subjects randomized into the intervention group then will undergo implantation of the bone-anchored anular closure prosthesis under fluoroscopic control and per the manufacturer's surgical technique and instructions for use. Subjects for whom the device is not successfully implanted will be considered treatment failures.

Parameters, such as duration of surgery, blood loss, length of hospitalization, and complications, will be recorded. Anteroposterior and neutral lateral radiographs will be obtained perioperatively for all randomized subjects. Immediate postoperative care, discharge, ambulation, and any physical therapy will be per hospital and investigator protocol.

\section{Device description}

The Barricaid $^{\circledR}$ (Intrinsic Therapeutics, Inc., Woburn, MA, USA) is an adjunct to lumbar discectomy designed to maintain the relative position of nucleus within the disc space. The device is comprised of a flexible polymer (polyethylene terephthalate (PET) or "Dacron") mesh that prevents migration of the nucleus from within the disc and a titanium (Ti6Al4V ELI) anchor that secures the mesh to one of the adjacent vertebral bodies 
as given in Figure 1. The mesh has a platinum iridium (radiopaque) marker for radiologic confirmation of mesh position and is attached to the anchor using suture. The implant is provided pre-assembled onto the delivery tool and has been CE-marked since 2009.

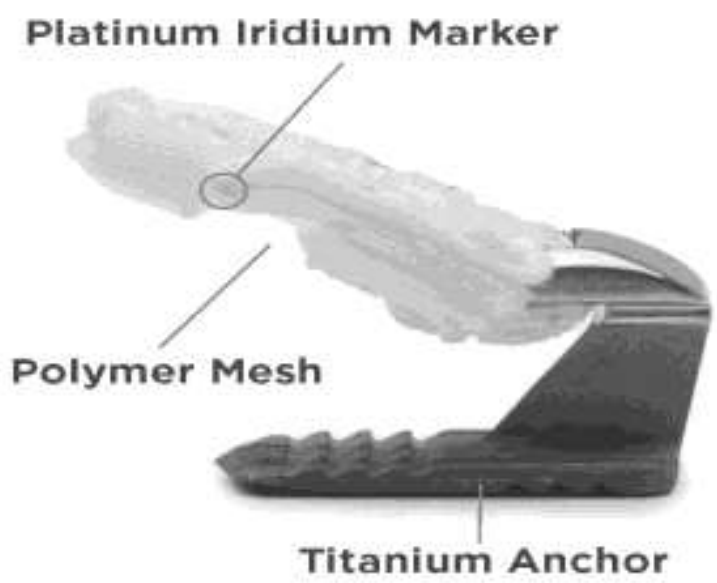

Figure 1: Graphic representation of the anular closure prosthesis, with a titanium bone anchor holding the polyester mesh in place.

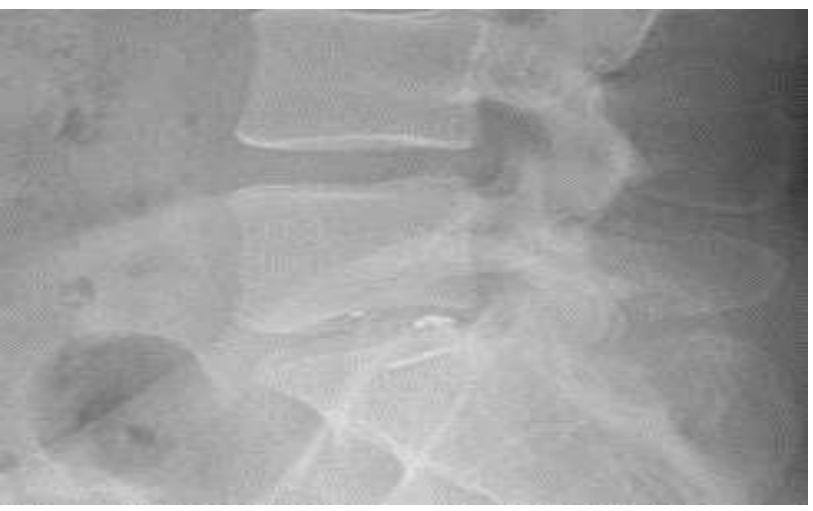

Figure 2: Neutral lateral radiograph obtained six weeks post-operatively showing the implanted device.

\section{RESULTS}

\section{Outcome assessment}

Current work status and pain medication intake, as well as clinical, neurologic, and radiologic assessments, will be obtained at all follow-up intervals per preoperative protocols. Postoperative CT and MRI per preoperative protocols will be obtained at all annual follow up intervals until the final patient enrolled reaches their 24 month follow-up.

\section{Independent radiologic analysis}

All radiologic imaging will be analyzed by independent certified radiologists. The specific parameters that will be assessed are summarized in Table 3 .
Table 3: Summary of radiologic evaluation parameters evaluated pre and postoperatively.

\begin{tabular}{|c|c|c|}
\hline $\begin{array}{l}\text { Radiologic Evaluation } \\
\text { Parameter }\end{array}$ & $\begin{array}{l}\text { Pre } \\
\text { operative }\end{array}$ & $\begin{array}{l}\text { Post } \\
\text { operative }\end{array}$ \\
\hline \multicolumn{3}{|l|}{ Quantitative measures } \\
\hline Disc angle & $\mathrm{X}$ & $\mathrm{X}$ \\
\hline $\begin{array}{l}\text { Angular motion (index and } \\
\text { adjacent) }\end{array}$ & $\mathrm{X}$ & $\mathrm{X}$ \\
\hline $\begin{array}{l}\text { Translational motion } \\
\text { (index and adjacent) }\end{array}$ & $\mathrm{X}$ & $\mathrm{X}$ \\
\hline $\begin{array}{l}\text { Disc height (index and } \\
\text { adjacent) }\end{array}$ & $\mathrm{X}$ & $\mathrm{X}$ \\
\hline $\begin{array}{l}\text { Change in disc height } \\
\text { (index and adjacent) }\end{array}$ & & $\mathrm{X}$ \\
\hline Spondylolisthesis & $\mathrm{X}$ & $\mathrm{X}$ \\
\hline $\begin{array}{l}\text { Change in } \\
\text { spondylolisthesis }\end{array}$ & & $\mathrm{X}$ \\
\hline \multicolumn{3}{|l|}{ Qualitative measurements } \\
\hline Heterotopic ossification & $\mathrm{X}$ & $\mathrm{X}$ \\
\hline $\begin{array}{l}\text { Osteophyte formation } \\
\text { (index and adjacent) }\end{array}$ & $\mathrm{X}$ & $\mathrm{X}$ \\
\hline Anular tears/fissures & $\mathrm{X}$ & $\mathrm{X}$ \\
\hline Disc signal intensity & $\mathrm{X}$ & $\mathrm{X}$ \\
\hline $\begin{array}{l}\text { Endplate changes/reactions } \\
\text { (MRI-based) }\end{array}$ & $\mathrm{X}$ & $\mathrm{X}$ \\
\hline $\begin{array}{l}\text { Endplate sclerosis (index } \\
\text { and adjacent) }\end{array}$ & $X$ & $\mathrm{X}$ \\
\hline Device condition & & $\mathrm{X}$ \\
\hline Device migration & & $\mathrm{X}$ \\
\hline Device subsidence & & $\mathrm{X}$ \\
\hline Reherniation & & $\mathrm{X}$ \\
\hline \multicolumn{3}{|c|}{ Quantitative and qualitative measurements } \\
\hline Modic change & $\mathrm{X}$ & $\mathrm{X}$ \\
\hline $\begin{array}{l}\text { Bone resorption (each } \\
\text { vertebral body, CT-based): } \\
\text { number of lesions and } \\
\text { lesion type }\end{array}$ & $\mathrm{X}$ & $\mathrm{X}$ \\
\hline Spontaneous fusion & & $\mathrm{X}$ \\
\hline
\end{tabular}

\section{Trial subgroups}

Single blinding subgroup

Investigational sites in the Netherlands will blind subjects (120 minimum) to their treatment arm as part of a singleblind cohort to assess any possible placebo effect. Such blinding is not possible in most other locations due to patient ownership of radiographic images. The investigators agree not to disclose randomization determination to the subject until completion of the study or subject withdrawal unless an emergency un-blinding is necessary. Accidental un-blinding will be documented, with continued monitoring per protocol. 


\section{Economic data subgroup}

Selected sites will collect economic data from subjects to augment healthcare utilization data from the study and to support a cost-effectiveness analysis. Subjects shall be consented to the additional data collection. Using cost diaries, subjects will report admissions to hospital, visits (specialists, general practitioner, physical therapy, and alternative health care), home care, paid domestic help, informal care, drugs and aids, and out-of-pocket expenses as a result of sciatica and/or back pain, as well as hours of absenteeism from work. Utilities represent the valuation of patient quality of life on a scale from 0 (as bad as death) to 1 (perfect health). The EuroQol classification system (EQ-5D), collected at the follow-up intervals established in the study protocol, and SF-6D utilities, calculated from the SF-36 data, will be used for societal valuation. $^{30-32}$
Patient valuation will be determined by transforming VAS and ODI scores to a utility scale. ${ }^{33}$ The total utility during each follow-up period will be calculated from the area under the utility curve as quality-adjusted life years (QALYs). Strict cost minimization analyses, comparing the costs of the two treatments, as well as cost utility analyses, comparing the cost per QALY gained/lost between the two groups, will be performed. Data will be analyzed by country, between countries, and across the entire data-set. A sample size of 150 subjects is anticipated for the cost analysis, but an interim analysis will be performed to determine if significance may be achieved earlier.

\section{Summary of study assessments and procedures}

The study assessments and procedures performed for each evaluation interval are outlined in Table 4.

Table 4: Outline of study assessments and procedures performed for each evaluation interval.

\begin{tabular}{|c|c|c|c|c|c|c|c|c|}
\hline Assessment/Procedure & Preoperative $^{\dagger}$ & Surgery & Follow & p exami & tions" & & & \\
\hline & & & $\begin{array}{l}6 \\
\text { weeks }\end{array}$ & $\begin{array}{l}3 \\
\text { months }\end{array}$ & $\begin{array}{l}6 \\
\text { months }\end{array}$ & $\begin{array}{l}12 \\
\text { months }\end{array}$ & $\begin{array}{l}24 \\
\text { months }\end{array}$ & $\begin{array}{l}\text { Additional } \\
\text { annual }\end{array}$ \\
\hline $\begin{array}{l}\text { Signed informed } \\
\text { consent/enrollment }\end{array}$ & $\mathrm{X}$ & & & & & & & \\
\hline $\begin{array}{l}\text { Demographic } \\
\text { information }\end{array}$ & $X$ & & & & & & & \\
\hline Medical history & $X$ & & & & & & & \\
\hline $\begin{array}{l}\text { Current pain } \\
\text { medication intake }\end{array}$ & $X$ & & $X$ & $X$ & $X$ & $X$ & $X$ & $\mathrm{X}$ \\
\hline Work status & $X$ & & $\mathrm{X}$ & $X$ & $X$ & $X$ & $\mathrm{X}$ & $X$ \\
\hline Clinical assessment & $X$ & & $\mathrm{X}$ & $X$ & $X$ & $X$ & $X$ & $\mathrm{X}$ \\
\hline $\begin{array}{l}\text { Neurological } \\
\text { evaluation }\end{array}$ & $X$ & & $X$ & $X$ & $X$ & $X$ & $X$ & $\mathrm{X}$ \\
\hline $\begin{array}{l}\text { Neutral Lateral and } \\
\text { AP Radiographs }\end{array}$ & X & X & X & X & $X$ & $X$ & $\mathrm{X}$ & $X$ \\
\hline $\begin{array}{l}\text { Flexion-extension } \\
\text { radiographs }\end{array}$ & $X$ & & & & & $X$ & $X$ & $X$ \\
\hline CT evaluation & $X$ & & & & & $X$ & $X$ & $\mathrm{X}$ \\
\hline MRI evaluation & $X$ & & & & & $X$ & $\mathrm{X}$ & $X$ \\
\hline $\begin{array}{l}\text { Patient } \\
\text { randomization }\end{array}$ & & $\mathrm{X}$ & & & & & & \\
\hline Perioperative details & & $\mathrm{X}$ & & & & & & \\
\hline $\begin{array}{l}\text { Economic data } \\
\text { (subgroup only) }\end{array}$ & & & $\mathrm{X}$ & $\mathrm{X}$ & $\mathrm{X}$ & $X$ & $\mathrm{X}$ & $\mathrm{X}$ \\
\hline
\end{tabular}

\section{Trial status}

Participants currently are being recruited for this trial.

\section{Safety considerations}

Adverse and serious adverse events: All adverse clinical events that occur during the study, having been absent at baseline, or were present at baseline and appear to worsen during the study, will be documented as AEs using definitions established by Good Clinical Practice (GCP) and the Food and Drug Administration (FDA) Guidelines. In addition to Institutional Review Board (IRB)/medical ethics committee (EC) reporting requirements, AEs will be reported to the sponsor within the following timeline requirements once the investigator 
learns of the event: all serious AEs, including subject deaths, within 24 hours; unanticipated adverse device effects within 24 hours; and all other AEs in a timely manner. The investigator must notify the respective IRB/EC of all unanticipated adverse device effects as soon as possible, but no later than 10 days after learning of the event.

\section{Subsequent surgical intervention}

Any surgical intervention performed at the treated level after the index surgery will be categorized as a revision (intervention group only), removal (intervention group only), supplemental fixation, or other reoperation. Reoperated subjects will remain in the study.

\section{Follow-up}

Subjects will be followed for at least 24 months, with routine follow-up examinations at six weeks; three, six, 12 , and 24 months; and annually thereafter until the last subject enrolled reaches the 24-month evaluation interval or the study is concluded. Besides the routine follow-up examinations conducted as part of the clinical trial, any adverse event that is related to the study and is continuing at the end of the study will be followed until the event has resolved or is determined to be irreversible.

\section{Data management and statistical analysis}

\section{Data management}

The study sponsor is responsible for data management, which involves an electronic data capture (EDC) system.

All data itemized in the trial protocol will be documented in the subjects' records and on provided source worksheets that follow standardized electronic case report forms (CRFs). Only authorized investigational site personnel will complete the source worksheets and electronic CRFs.

Electronic CRFs must be reviewed and approved by an investigator. Since there is a potential for errors, inaccuracies, and misinterpretation in transcribing data from source documents into the EDC system, originals or photocopies of all relevant worksheets, records and reports, and copies of test results must be available at all times for inspection and comparison to the electronic data capture data by the study monitor.

The study sponsor is responsible for database development and data acquisition, storage, and validation. Data validation involves controls of completeness, consistency, and plausibility of data documented on CRFs using a query system between data management and investigators. After resolution of queries and upon trial closure, the database will be closed and forwarded to a biostatistician for analysis. Following completion of this trial, final reports will be issued as required.

\section{Sample size calculation}

A Bayesian approach to sample size selection will be used. A minimum total sample size of 400 and a maximum of 800 will be considered. An interim analysis will be performed when 400 subjects have been accrued. If trial success is determined to be highly likely, per the statistical analysis plan, then accrual will be stopped. If accrual continues, another interim analysis will be performed after 50 additional subjects have been accrued. These 100-subject incremental analyses will continue until accrual is stopped or 800 subjects have been accrued.

\section{Statistical analysis}

\section{Interim analyses}

The interim analyses performed before patient enrollment is stopped will be conducted as sample size determination analyses. The joint predictive probability of superiority for both co-primary endpoints will be calculated. All interim results available will be used to calculate the predictive probability of trial success for the currently accrued subjects. A decision then will be made to stop the trial at the current sample size or to continue enrollment.

\section{Early claims interim analyses}

After the sample size is determined, early claim interim analyses will be performed. These analyses will occur when accrual is stopped and at 6,12, and 18 months after accrual is stopped, yielding a possible total of four early claim analyses. No early claim analysis will be performed until at least 200 intervention group subjects have reached the 24 month follow-up interval. If the predictive probability of success (joint probability for each endpoint) in an early claim analysis is at least 0.99 , then an immediate claim of superiority will be made.

\section{Primary statistical analysis}

For each of the two co-primary endpoints, the claim of superiority will be accepted if the posterior probability of superiority is larger than 0.95 .

The design of the mesh was modified slightly toward the beginning of the study (after 45 intervention group subjects had been enrolled). This design change was implemented to strengthen the attachment of the mesh to the anchor in response to a small number of failures $(<1 \%)$ in the commercial experience. At trial completion, a logistic regression model that includes terms for time, treatment, and device generation will be used to determine if the treatment effect varies by generation of device. If this interaction is significantly different at the $5 \%$ level for either co-primary endpoint, then the modified intent-to-treat (mITT) population will include all subjects randomized to the control group and all subjects randomized to the treatment group who received 
the current generation device. If no statistically significant interaction is found, then the mITT population will include all randomized subjects. Subjects will be classified by the group in which they are randomized, regardless of treatment (or generation device) received.

The primary analysis will be performed on the mITT population. Subjects with missing data will be included in this primary analysis using Bayesian multiple imputation. This approach will enable an analysis to be performed based on the full mITT population.

\section{Safety analyses}

For each safety analysis, the outcome will be an event (possible composite). Traditional frequentist confidence intervals and p-values will be used.

\section{Site heterogeneity}

Analyses of data homogeneity between sites will be performed for each co-primary endpoint and number of AEs. Sites with less than five subjects will be combined to one mega site. If the results of the test show evidence of lack of heterogeneity, then a hierarchical model will be created to model the success rate per site as the hierarchical component.

\section{Subgroup analyses}

The results of the subgroup of subjects who were blinded to the received treatment will be included in the overall study population determinations. Effectiveness analyses also will be conducted, based on the mITT population, in each of these subject subgroups (blinded and un-blinded).

For the cost-utility analysis, both strict cost minimization analyses, comparing the costs of the two treatments, and cost utility analyses, comparing the cost per QALY gained with the adjunctive use of the prosthesis for anular closure (if any) with limited discectomy alone, will be performed.

\section{Quality assurance}

The study protocol was approved at each investigational site by the local IRB or EC. The study is being conducted in accordance with GCP and all applicable local and national regulatory and institutional requirements, including those for subject privacy and informed consent, the local institutional boards or medical ethics committees of all investigational sites, the FDA guidelines for the conduct of clinical trials, each country's competent authority when applicable, and International Community on Harmonization (ICH) and ISO 14155 guidelines, as these pertain to the control and conduct of clinical trials.

The study sponsor arranges for regular inspection of all study records, including CRFs, source documents, and regulatory documents during the study by a monitor.
Such inspection is performed to ensure that the study is conducted and documented in accordance with federal regulations and the terms of the protocol. Investigators also must agree to allow inspections by staff members of the FDA or other regulatory agencies before, during, or after the study has concluded, if such inspections are requested.

The trial also will be monitored by an independent DSMB comprised of physicians in the fields of radiology and neurological, orthopedic, and spine surgery who are independent of the study sponsor and the sites. The DSMB will review accumulating safety-related issues, including all AEs and protocol deviations, on at least a quarterly basis and advise the study sponsor regarding the continued safety of the current study population and those yet to be recruited. The sponsor must report serious adverse events (SAEs) to the DSMB as they occur. At any time, the DSMB may recommend stoppage of the trial. The trial may be terminated based on the predetermined stopping rules defined in the protocol as in Table 5, but also may be stopped at the discretion of the DSMB for any reason related to safety or ethics.

\section{Expected outcomes of the study}

It is hypothesized that augmentation with the boneanchored prosthesis for anular closure leads to improved outcomes, including reduced pain, dysfunction, and herniation recurrence, at a follow-up of at least 24 months. Through rigorous studies such as this one, the outcome of spinal procedures and the impact of new technologies may be adequately assessed. The resulting level I evidence will provide an initial understanding of the advantages and disadvantages of this device. This type of evidence improves the decision-making process of patients, surgeons, and healthcare institutions in selecting effective treatment for lumbar disc herniation. If this hypothesis is correct and the trial demonstrates that augmentation of traditional discectomy with the boneanchored prosthesis for anular closure leads to improved mid-term outcomes, including reduced pain, dysfunction, and herniation recurrence, then use of this device in conjunction with limited discectomy should be recommended and additional longer-term study may be warranted.

\section{Duration of the project}

Study recruitment is anticipated to take approximately three years. After follow-up of at least 24 months is reached on all subjects and upon trial closure, the process of finalizing and analysing the data will begin. The goal for completing a manuscript based on the 24-month evaluations is six months after trial closure. Enrollment has been sucessfully completed with 550 patients.

\section{Project management}

The organizational infrastructure of the study includes the study sponsor's regulatory and clinical department, 
tasked with overseeing training, data monitoring, DSMB communication, and site coordination; a DSMB, which oversees overall study safety and ethics and resolves data- or safety-related disputes; the lead investigator at each site, responsible for patient recruitment, safety, treatment, and follow-up evaluations; other investigators, to whom study tasks such as patient recruitment, treatment, and follow-up evaluations may be formally delegated; and study coordinators, who may be formally designated to recruit and consent patients and coordinate follow-up under the supervision of the investigator.

\section{Ethics}

\section{Informed consent}

Informed consent is obtained from all potential study participants using the approved informed consent form (ICF). The investigator or a person designated by the investigator who acts under the investigator's responsibility, informs the potential study participant of all pertinent aspects of the study. The study and informed consent form are discussed in a language and in terms that each possible participant is able to understand. Patients also are informed that their medical care will not be affected should they elect not to participate. Documentation that the ICF was signed and dated prior to any study procedure is made at the time of the informed consent and is filed as a source document at the investigational site. A copy of the ICF is given to the study subject.

\section{Study risks}

Patient reported and investigator documented outcomes may be biased by a lack of blinding to treatment group. For subjects in the intervention group, the risks associated with the surgical procedure are identical to that of a standard lumbar discectomy until completion of the discectomy. After discectomy is completed, the devicerelated instrumentation is introduced. Risks associated with the device include device migration, fracture and subsidence; foreign body reaction or allergic material reaction; and possibly an increased risk of infection, dural tear or other neurologic deficiency. These risks are theoretically mitigated by a decreased risk of reherniation. A full risk analysis including the results of ongoing prospective studies with the bone-anchored prosthesis for anular closure was performed and presented to the investigators. Safety related issues that occur both within the clinical trial, as well as in ongoing commercial activity, are monitored closely by the study sponsor.

Table 5: Study suspension criteria requiring trial suspension.

\begin{tabular}{|c|c|}
\hline Event & $\begin{array}{l}\text { dy Suspension Criteria } \\
\text { tervention Group Only) }\end{array}$ \\
\hline $\begin{array}{l}\text { Reoperation rate of device- or } \\
\text { procedure-related reoperations } \\
\text { (for revision, removal, } \\
\text { supplemental fixation, or } \\
\text { reherniation) }\end{array}$ & $\begin{array}{l}\text { - At least three observed occurrences in the intervention group; and } \\
\text { the percentage of patients experiencing reoperation is more than } 10 \% \\
\text { higher in the intervention group compared with the control group, or is } \\
\text { more than } 25 \% \text { higher in the intervention group absolutely. }\end{array}$ \\
\hline Device removal rate & $\begin{array}{l}\text { - At least three observed occurrences; and } \\
\text { the percentage of subjects experiencing device removal is more than } 15 \% \\
\text { of the intervention group. }\end{array}$ \\
\hline Implant integrity & $\begin{array}{l}\text { - At least three observed occurrences; and } \\
\text { the percentage of patients experiencing loss of implant integrity, } \\
\text { including device breakage, fracture, or device loosening, is more than } \\
15 \% \text { of the intervention group. }\end{array}$ \\
\hline Neurological adverse events & $\begin{array}{l}\text { - At least three observed occurrences in the intervention group; and } \\
\text { the percentage of patients experiencing serious device- or procedure- } \\
\text { related neurological events is more than } 10 \% \text { higher in the intervention } \\
\text { group compared with the control group, or is more than } 15 \% \text { higher in the } \\
\text { intervention group absolutely. }\end{array}$ \\
\hline Spontaneous fusion & $\begin{array}{l}\text { - At least three observed occurrences in the intervention group; and } \\
\text { the percentage of patients experiencing unintended fusion is more than } \\
10 \% \text { higher in the intervention group compared with the control group, or } \\
\text { is more than } 15 \% \text { in the intervention group absolutely. }\end{array}$ \\
\hline Infection & $\begin{array}{l}\text { - At least three observed occurrences in the intervention group; and } \\
\text { the percentage of patients experiencing a deep wound infection in the } \\
\text { intervention group is more than } 10 \% \text { of the intervention group. }\end{array}$ \\
\hline
\end{tabular}




\section{DISCUSSION}

Aside from the early experimentation with the Fernstrom ball and the suturing techniques of Yasargil, mechanical nucleus replacement or augmentation devices have been used in Europe for over 20 years and prostheses for anular closure have been used for almost 10 years. ${ }^{34-41}$ Preliminary results of most designs involved small series and were not sufficiently safe to warrant larger clinical series, leading to device discontinuation, redesign, or premature commercial launch. ${ }^{40,41}$ The overall conclusion from these experiences has been that materials inserted into the disc will migrate, extrude, or subside into the endplates if not firmly anchored to the bone. ${ }^{35,37,38,40,41}$

The objective of the current trial is to demonstrate that a prosthesis for anular closure can safely and effectively prevent poor outcomes after discectomy, namely, recurrent pain, dysfunction, and same-level herniation, by blocking the anular defect. Building upon lessons learned from previous designs, the current design includes an anchor that secures the occlusion component to an adjacent vertebral body in an effort to minimize migration and subsidence. The device is designed to be used in conjunction with a limited discectomy, which retains as much nuclear material as possible to maintain disc height and avoid the onset of new, mechanical low back pain, a typical problem associated with aggressive discectomies. $^{7,21,42}$ This bone-anchored prosthesis for anular closure should prevent recurrent herniation, a common complication associated with conservative discectomies. ${ }^{7,21,42}$ This novel design is being evaluated through a rigorous multicenter, prospective, randomized control superiority trial that will enable comparison of pre-, intra-, and postoperative data, including standardized self-reported and surgeon-assessed clinical outcomes, an independent radiologic analysis, and a costutility analysis. To our knowledge, not only is this the first randomized, controlled trial evaluating a boneanchored prosthesis for anular closure, but it likely also is the largest multi-country, prospective discectomy study performed to date.

Randomized controlled trials designed to establish superiority are considered to be the gold standard in clinical trial research. ${ }^{43}$ To minimize procedural variability on outcome, the Spengler technique for limited discectomy will be performed for all subjects. ${ }^{29}$ Thus, the control group will undergo standard of care discectomy and the intervention group will undergo the same type of discectomy, augmented with the bone-anchored prosthesis for anular closure. Because patients with very small "fissure" defects have a documented low risk of reherniation, they likely would not benefit from implantation of this type of device when used with a limited discectomy and, therefore, were excluded from participation. ${ }^{18}$

Twenty-four months was chosen for study evaluation based on prevailing regulatory guidance regarding safety evaluation of spinal systems and because most clinical improvement from surgery to treat lumbar disc herniation has been shown to occur well within this time period. ${ }^{44,45}$ The effectiveness component of the success criteria is based on results from previous studies. Beurskens et al reported that a change of 4 to 6 points in the 100-point ODI scale was necessary to represent a clinically significant improvement in patients with low back pain. ${ }^{46}$ To be conservative, an improvement of at least 15 points in ODI was selected for this study. The 20-point improvement in VAS leg pain scores necessary to establish success in this trial is based on a study examining clinically relevant VAS pain score changes in patients with acute pain caused by rheumatic conditions. $^{47}$ In a retrospective analysis of long-term outcomes (defined as greater than 10 years) of lumbar discectomy, Yorimitsu et al documented that long-term low back pain scores were significantly lower, corresponding to worse pain, in patients who lost more than $25 \%$ of preoperative disc height. ${ }^{5}$ Based on these results, subjects in the current trial must maintain a postoperative average disc height $75 \%$ or greater than preoperative disc height to be considered a success.

An ITT statistical analysis was selected for study group comparison because it implies a conservative effect on trial outcome; therefore, if the study is conducted poorly, then it will be unlikely that the intervention will be proven to be more effective than the control. ${ }^{43}$

\section{CONCLUSION}

The analyses described in the protocol will yield information about device performance. Effectiveness analyses comparing results between the blinded and unblinded subgroups will assess a possible placebo effect associated with use of the device. The cost-effectiveness analysis is of critical significance, as it will evaluate the trade-off between improved clinical outcomes and quality of life on the one hand, and the societal costs associated with treatment, follow-up visits, and patient recovery on the other. This type of analysis is becoming increasingly important as governments and health insurers continue to be pressured to spend limited healthcare funding wisely.

\section{ACKNOWLEDGMENTS}

We acknowledge Jodi F. Hartman, MS, for editorial assistance with the manuscript.

Funding: Financial support given by Intrinsic therapeutics provided Conflict of interest: None declared

Ethical approval: The study was approved by the Institutional Ethics Committee

\section{REFERENCES}

1. Garg B, Nagraja UB, Jayaswal A. Microendoscopic versus open discectomy for lumbar disc herniation: 
a prospective randomised study. J Orthop Surg. 2011;19:30-4.

2. Gibson JN, Waddell G. Surgical interventions for lumbar disc prolapse: updated Cochrane Review. Spine. 2007;32:1735-47.

3. Mariconda M, Galasso O, Secondulfo V, Rotonda GD, Milano C. Minimum 25-year outcome and functional assessment of lumbar discectomy. Spine. 2006;31:2593-9.

4. Nyström B. Experience of microsurgical compared with conventional technique in lumbar disc operations. Acta Neurol Scand. 1987;76:129-41.

5. Yorimitsu E, Chiba K, Toyama Y, Hirabayashi K. Long-term outcomes of standard discectomy for lumbar disc herniation: a follow-up study of more than 10 years. Spine. 2001;26:652-7.

6. Carragee EJ, Han MY, Yang B, Kim DH, Kraemer $\mathrm{H}$, Billys J. Activity restrictions after posterior lumbar discectomy. A prospective study of outcomes in 152 cases with no postoperative restrictions. Spine. 1999;24:2346-51.

7. Carragee EJ, Spinnickie AO, Alamin TF, Paragioudakis S. Carragee EJ, Spinnickie AO, et al. A prospective controlled study of limited versus subtotal posterior discectomy: short-term outcomes in patients with herniated lumbar intervertebral discs and large posterior anular defect. Spine. 2006;31:653-7.

8. Caspar W, Campbell B, Barbier DD, Kretschmmer R, Gotfried Y. The Caspar microsurgical discectomy and comparison with a conventional standard lumbar disc procedure. Neurosurgery. 1991;28:78-87.

9. Barth M, Diepers M, Weiss C, Thomé C. Two-year outcome after lumbar microdiscectomy versus microscopic sequestrectomy: part 2: radiographic evaluation and correlation with clinical outcome. Spine. 2008;33:273-9.

10. Barth M, Weiss C, Thomé C. Two-year outcome after lumbar microdiscectomy versus microscopic sequestrectomy: part 1: evaluation of clinical outcome. Spine. 2008;33:265-72.

11. Fu TS, Lai PL, Tsai TT, Niu CC, Chen LH, Chen WJ. Long-term results of disc excision for recurrent lumbar disc herniation with or without posterolateral fusion. Spine. 2005;30:2830-4.

12. Loupasis GA, Stamos K, Katonis PG, Sapkas G, Korres DS, Hartofilakidis G. Seven- to 20-year outcome of lumbar discectomy. Spine. 1999;24:2313-7.

13. McGirt MJ, Eustacchio S, Varga P, Vilendecic M, Trummer M, Gorensek M, et al. A prospective cohort study of close interval computed tomography and magnetic resonance imaging after primary lumbar discectomy: factors associated with recurrent disc herniation and disc height loss. Spine. 2009;34:2044-51.

14. Suk KS, Lee HM, Moon SH, Kim NH. Recurrent lumbar disc herniation: results of operative management. Spine. 2001;26:672-6.
15. Thomé C, Barth M, Scharf J, Schmiedek P. Outcome after lumbar sequestrectomy compared with microdiscectomy: a prospective randomized study. J Neurosurg Spine. 2005;2:271-8.

16. Ambrossi GL, McGirt MJ, Sciubba DM, Witham TF, Wolinsky JP, Gokaslan ZL, et al. Recurrent lumbar disc herniation after single-level lumbar discectomy: incidence and health care cost analysis. Neurosurgery. 2009;65:574-8.

17. Arts MP, Brand R, van den Akker ME, Koes BW, Bartels RH, Peul WC. Leiden-The Hague Spine Intervention Prognostic Study Group (SIPS) Tubular diskectomy vs conventional microdiskectomy for sciatica: a randomized controlled trial. JAMA. 2009;302:149-58.

18. Carragee EJ, Han MY, Suen PW, Kim D. Clinical outcomes after lumbar discectomy for sciatica: the effects of fragment type and anular competence. J Bone Joint Surg Am. 2003;85:102-8.

19. Fountas KN, Kapsalaki EZ, Feltes CH, Smisson HF $3^{\text {rd }}$, Johnston KW, Vogel RL, et al. Correlation of the amount of disc removed in a lumbar microdiscectomy with long-term outcome. Spine. 2004;29:2521-6.

20. Gaston P, Marshall RW. Survival analysis is a better estimate of recurrent disc herniation. J Bone Joint Surg Br. 2003;85:535-7.

21. McGirt MJ, Ambrossi GL, Datoo G, Sciubba DM, Witham TF, Wolinsky JP, et al. Recurrent disc herniation and long-term back pain after primary lumbar discectomy: review of outcomes reported for limited versus aggressive disc removal. Neurosurgery. 2009;64:338-45.

22. Rogers LA. Experience with limited versus extensive disc removal in patients undergoing microsurgical operations for ruptured lumbar discs. Neurosurgery. 1988;22:82-5.

23. Weinstein JN, Lurie JD, Tosteson TD, Skinner JS, Hanscom B, Tosteson AN, et al. Surgical vs nonoperative treatment for lumbar disk herniation: the Spine Patient Outcomes Research Trial (SPORT) observational cohort. JAMA. 2006;296:2451-9.

24. Cadarette SM, Jaglal SB, Murray TM. Validation of the simple calculated osteoporosis risk estimation (SCORE) for patient selection for bone densitometry. Osteoporos Int. 1999;10:85-90.

25. Lydick E, Cook K, Turpin J, Melton M, Stine R, Byrnes C. Development and validation of a simple questionnaire to facilitate identification of women likely to have low bone density. Am J Manag Care. 1998;4:37-48.

26. Fairbank RCT, Pynsent PB. The Oswestry disability index. Spine. 2000;25:2940-53.

27. Ware JE, Kosinski M, Dewey JE. How to Score Version 2 of the SF-36 ${ }^{\circledR}$ Health Survey. QualityMetric Incorporated; Lincoln, RI.: 2000.

28. Ware JE, Koskinski M, Keller SD. SF-36® Physical and Mental Health Summary Scales: A User's 
Manual. Boston, Massachusetts, The Health Institute; 1994

29. Spengler DM. Lumbar discectomy. Results with limited disc excision and selective foraminotomy. Spine. 1982;7:604-7.

30. Dolan P. Modeling valuations for the EuroQol health states. Med Care. 1997;35:1095-108.

31. Lamers LM, Stalmeier PF, McDonnell J, Krabbe $\mathrm{PF}$, van Busschbach JJ. Measuring the quality of life in economic evaluations: the Dutch EQ-5D tariff [in Dutch]. Ned Tijdschr Geneeskd. 2005;149:1574-8.

32. Brazier J, Roberts J, Deverill M. The estimation of a preference-based measure of health from the SF-36. J Health Economics. 2002;21:271-92.

33. Carreon LY, Glassman SD, McDonough CM, Rampersaud R, Berven S, Shainline M. Predicting SF-6D utility scores from the Oswestry disability index and numeric rating scales for back and leg pain. Spine. 2009;34:2085-9.

34. Fernstrom U. Arthroplasty with intercorporal endoprosthesis in herniated disc and in painful disc. Acta Chir Scand Suppl. 1966;357:154-9.

35. Siemionow KB, Hu X, Lieberman IH. The Fernstrom ball revisited. Eur Spine J. 2012;21:4438.

36. Yasargil MG. Microsurgical operation of herniated lumbar disc. Adv Neurosurg. 1977;4:81-2.

37. Bao QB, Yuan HA. Artificial disc technology. Neurosurg Focus. 2007;9:14.

38. Coric D, Mummaneni PV. Nucleus replacement technologies. J Neurosurg Spine. 2008;8:115-20.

39. Husson JL, Korge A, Polard JL, Nydegger T, Kneubuhler S, Mayer HM. A memory coiling spiral as nucleus pulposus prosthesis: concept specifications, bench testing, and first clinical results. J Spinal Disord Tech. 2003;16:405-11.

40. Klara PM, Ray CD. Artificial nucleus replacement: clinical experience. Spine. 2002;27:1374-7.
41. Trummer M, Eustacchio S. Design history of an anular reconstruction device: from failure to success. Eur Spine J. 2010;19:2051.

42. Watters WC 3rd, McGirt MJ. An evidence-based review of the literature on the consequences of conservative versus aggressive discectomy for the treatment of primary disc herniation with radiculopathy. Spine. 2009;9:240-57.

43. Lesaffre E. Superiority, equivalence, and noninferiority trials. Bull NYU Hosp Jt Dis. 2008;66:150-4.

44. United States Department of Health and Human Services Food and Drug Administration Center for Devices and Radiological Health. Guidance Document for the Preparation of IDEs for Spinal Systems. Document issued on: January 13, 2000. Retrieved August 1, 2012 from http://www.fda.gov/MedicalDevices/DeviceRegulati onandGuidance/GuidanceDocuments/ucm073771.ht $\mathrm{m}$.

45. Atlas SJ, Keller RB, Wu YA, Deyo RA, Singer DE. Long-term outcomes of surgical and nonsurgical management of sciatica secondary to a lumbar disc herniation: 10 year results from the Maine Lumber Spine Study. Spine. 2005;30:927-35.

46. Beurskens AJ, de Vet HC, Koke AJ. Responsiveness of functional status in low back pain: a comparison of different instruments. Pain. 1996;65:71-6.

47. Grilo RM, Treves R, Preux PM, Vergne-Salle P, Bertin P. Clinically relevant VAS pain score change in patients with acute rheumatic conditions. Joint Bone Spine. 2007;74:358-61.

Cite this article as: Klassen PD, Hes R, Bouma GJ, Eustacchio S, Barth M, Kursumovic A, et al. A multicenter, prospective, randomized study protocol to demonstrate the superiority of a bone-anchored prosthesis for anular closure used in conjunction with limited discectomy to limited discectomy alone for primary lumbar disc herniation. Int J Clin Trials 2016;3(3):120-31. 